\title{
Power Network Reliability Evaluation Framework Considering OHL Electro-Thermal Design
}

\author{
K. Kopsidas, Member, IEEE, C. Tumelo-Chakonta, Student Member, IEEE, and C. Cruzat, Student \\ Member, IEEE
}

\begin{abstract}
Utilities continually investigate ways to optimize the utilization of their existing plant and improve their network's flexibility and resiliency to future uncertainties. With respect to overhead lines (OHL), smart grid solutions aim to increase adequacy through the implementation of probabilistic thermal rating (PTR), dynamic thermal rating (DTR) or novel high temperature low sag (HTLS) conductor technologies. At present, the risks related to these particular solutions, both for the OHL plant and the power network, are not quantified. This paper presents a novel methodology for power network reliability evaluation which integrates a network level sequential Monte Carlo algorithm with a detailed modelling of OHL. This integration facilitates a holistic evaluation of power network reliability as it considers the properties of $\mathrm{OHL}$ design technologies and their associated ageing risks. As a result, the network performance (adequacy) and plant risks (ageing) introduced by increased OHL capacities through PTR, DTR and HTLS solutions can be objectively quantified. An application of the methodology is demonstrated using the IEEE-RTS 96 network which is assessed considering PTR-based OHL capacities.
\end{abstract}

Index Terms-Ageing, ampacity, asset management, sequential Monte Carlo, overhead line, power system planning, probabilistic methods, reliability assessment.

\section{INTRODUCTION AND BACKGROUND}

$\mathbf{U}$ TILITIES are under pressure to realize and implement overhead line (OHL) network expansion solutions that aim to reliably connect increasing capacities of renewable energy sources. However, in reaction to regulatory resistances to build new OHLs, utilities are challenged to improve network performance through increasing utilization of existing OHL assets. As a result, flexible alternative current transmission system (FACTS) solutions have been engineered to increase network power flow adequacies by maintaining network voltages within acceptable levels; this is achieved through relaxing the electrical constraints of existing OHLs [1]. However, in other cases the increase in network power transfer adequacies is achieved by relaxing the thermal, rather than the electrical, constraints of particular OHLs [2].

Inexpensive methods engineered to overcome thermal rating constraints are based on probabilistic thermal rating (PTR) or on dynamic thermal rating (DTR) concepts [3-6]. The PTR considers historical weather values and a constant high OHL power flow in order to establish a probability of exceeding the

Konstantinos Kopsidas (k.kopsidas@manchester.ac.uk), Chomba TumeloChakonta (chomba.tumelo-chakonta@postgrad.manchester.ac.uk) and Carlos Cruzat (carlos.cruzathermosilla@manchester.ac.uk) are with the School of Electrical and Electronic Engineering, University of Manchester, UK. design temperature. Increasing this probability (excursion time) increases the line's adequacy but also the risk of overloading (i.e., exceeding the design temperature) and therefore a line's risk of accelerated ageing [7]. DTR is based on monitoring the actual weather conditions and conductor properties to evaluate OHL thermal ratings in real time $[1,8-10]$. When compared to PTR, DTR has a smaller conductor risk of overloading and accelerated ageing. When further increase in OHL adequacy is required more expensive solutions that involve re-tensioning or reconductoring with High Temperature Low Sag (HTLS) conductors can also be considered. These are also low ageing risk uprating methods, since the conductors are engineered with improved materials capable of enduring high operating temperatures which conventional conductors cannot endure [11, 12]. These options are less popular among utilities as they also require interruption of the OHL, however their additional benefit on ageing resistance operation is not yet evaluated.

The current preference to increase existing OHL networks adequacies through affordable PTR methods led utilities to increase excursion times, reducing in this way any initial sufficient thermal safety margins within which they used to operate, without considering the consequences of such actions on OHL accelerated ageing risk [13]. This could be the reason for infrequent but expensive permeated risks relating to network performance and occasional blackouts [14, 15].

Quantifying the risk of ageing for the different methods of increasing OHL network adequacy is thus important in order to evaluate both inexpensive (PTR and extensive emergency operation durations) and more expensive solutions. This could justify the implementation of more 'expensive' solutions which reduce this ageing risk and increase overall network performance. Such solutions could involve investments in technologies that are resistant to accelerated ageing (i.e., FACTS, DTR monitoring equipment, HTLS conductors) or maintenance/repairing actions (i.e., re-tensioning/reconductoring OHL sections, maintenance of right-of-way) [15]. In order to holistically evaluate the potential benefits of such technologies, reliability network assessments should be performed to quantify their risk of ageing and its impact on the overall network performance.

Existing methods for reliability evaluation of power systems employ either deterministic or probabilistic techniques [16]. Deterministic methods are based upon an engineering judgment criterion that validates a system as reliable if failures of a specified number of system plants do not result in system failure. This is expressed as $\mathrm{N}-n$ where $n$ specifies the number of plants that can fail without affecting the system's integrity. 
Most system analyses are performed with $n$ being either 1or 2 [17]. However, deterministic methods do not account for the true stochastic nature of network component failures. Thus, probabilistic methods are used to evaluate network reliability more accurately by utilizing a variety of measurable indices that provide understanding and practical quantification of network operational risks [16, 18-22]. The most commonly used probabilistic network assessment methods are mainly based on the Monte Carlo Simulation usually modelled in three variants: State Based, State Transition and Sequential Monte Carlo (SMC) simulations. A thorough discussion of these methods is given in [23-25]. However, relatively little work has incorporated electro-thermal modelling into the reliability framework [26, 27]. Moreover, these studies fail to consider the effect of emergency operation and the associated impact on OHL conductor ageing. Thus they cannot be used to evaluate benefits and risk of ageing prone and ageing free technologies.

Since utilities are currently forced to economically increase the utilization and efficiency of their existing OHL network infrastructure they continuously investigate methods to operate their OHLs at higher temperatures (i.e., hot-wiring) [28]. However, very little research has been done to assess the associated risk of thermal ageing and the benefits from increased utilization for each OHL within a network-wide analysis. To facilitate such evaluation, this paper proposes a novel power network reliability assessment methodology which integrates OHL design properties describing the network's electro-thermal conditions. This allows for the risk related with operational (e.g. extended emergency ratings) and design (e.g. increased PTR) decisions to be assessed. Such methodology will enable identifying the cases where an increased utilization of OHLs results in increased benefit in the network particularly when novel technologies and smart energy solutions could contribute to network flexibility and risk resiliency and thus be more comprehensively evaluated.

This novel reliability evaluation framework is detailed in the next section, followed by one example application.

\section{Reliability EVALUATION FraMEWORK CONSIDERING OHL ELECTRO-THERMAL DESIGN PROPERTIES}

Every OHL within a network experiences different operating conditions due to different numbers of failures, post-contingent loading events and ambient weather conditions [29]. Consequently, each OHL experiences a particular operating temperature $T_{c}$ at time $t$ with a probability of $P\left(T_{c}(t)\right)$ that is determined by the OHL's probability to experience a maximum current capacity of $P\left(I_{c, \max }(t)\right)$ and a current flow of $P\left(I_{\text {flow }}(t)\right)$ at the same time $t$, as described by (1).

$$
P\left(T_{c}(t)\right)=P\left(I_{c, \text { max }}(t)\right) * P\left(I_{\text {flow }}(t)\right)
$$

In (1) $P\left(I_{\text {flow }}(t)\right)$ is a system dependent probability which results from the stochastic combination of plant failures, intermittent generation and power demand. In the same equation, $P\left(I_{c, \max }(t)\right)$ is an OHL dependent probability which results from the joint probabilities of weather conditions and the OHL conductor resistance, $R\left(T_{c, \max }\right)$, at the maximum operating temperature, $T_{c, \max }$, and its structural properties. The maximum
OHL continuous current rating, $I_{c, \max }(t)$, is therefore a function of time, and for a given instance, $t$, it is calculated by (2) [30].

$$
I_{c, \text { max }}(t)=\sqrt{\frac{Q_{c}\left(V_{w}(t), T_{a}(t), T_{c, \text { max }}\right)+Q_{r}\left(T_{a}(t), T_{c, \text { max }}\right)-Q_{s}(t)}{R\left(T_{c, \text { max }}\right)}}(2)
$$

Where, $Q_{c}\left(V_{w}(t), T_{a}(t), T_{c, \max }\right)$ is the convection heat loss depending on the conductor geometry, wind speed $V_{w}(t)$, ambient temperature $T_{a}(t)$, and $T_{c, \max } . Q_{r}\left(T_{a}(t), T_{c, \max }\right)$ is the radiation heat loss and $Q_{s}(t)$ is the conductor solar heat gain.

Since $P\left(T_{c}(t)\right)$ describes the OHL operational state it can, then, be used to identify the OHL's thermal operation profile and therefore its ageing due to this thermal profile [31]. The challenge is to consider these profiles at the network level where additional failures affect the profiles and ageing risks. A framework to address this is presented next.

\section{A. Outline of the computational framework}

The proposed framework is outlined in Fig.1 with input data describing the plant as well as the network characteristics feeding into the system status initialization block. In this block the minimum transition time-step, $\Delta t$, is specified as the smallest transition time of either the OHL weather or the power demand (this affects the accuracy of the analysis and the speed of computations). The weather data of each OHL are time-stamped to be 'synchronized' with the network demand data at every $\Delta t$. Additionally, the system status initialization block performs a single AC power flow (ACPF) in order to set the initial system state $(t=0)$ and identify violated thermal constraints.

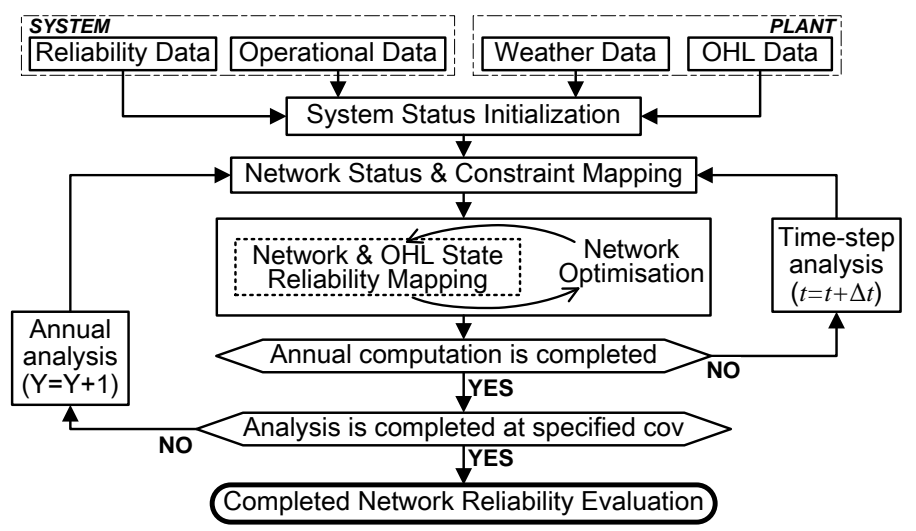

Fig. 1. Flowchart of the computations framework.

The system analyses are then performed through two distinct iterative computational loops. One is implemented for a $\Delta t$ timestep to determine changes on both plant and network status, while the other loop determines the annual reliability computations. This complete computational process is divided into the 'network status \& constraint mapping', as well as the 'network optimization' and 'network \& OHL state reliability mapping' blocks shown in Fig.1. The network status \& constraint mapping evaluates the adequacy of each OHL of the network by considering reliability, operational, weather and OHL design data and maps the network topology by identifying each component's operation state. The operation and restoration transition histories of the components are generated using a 
SMC algorithm which also considers, in addition to demand changes, the weather changes in every $\Delta t$ and consequently, the exceedance time of OHLs thermal constrains. Thus, within the network status \& constraint mapping block, an additional small computational feedback is implemented to capture cascading failures, if any, which occur at the same $\Delta t$ duration due to thermal exceedance events. This feedback loop is applied until the network status \& constraint mapping is fully updated by realising all possible cascading events which have occurred at the same $\Delta t$ duration when thermal exceedances are present.

The network status \& constraint mapping output then feeds into the network optimisation, in which ACPF computations are initially performed to identify network constraints. When either a voltage or a thermal constraint is present, an AC optimal power flow is performed considering corrective re-dispatching and load shedding actions to minimise the total operation cost. Only static system constraints are considered, while dynamic/transient system constraints are neglected to relieve computational burdens, but can be included if desired.

Within the network optimisation block the network \& OHL state reliability mapping is performed (Fig. 1) by utilising results from the network optimisation to update the calculated indices required to capture the cost of network operation. The indices are divided into two categories: 1) those which describe the complete network performance, namely, the expected energy not served (EENS), expected frequency of load curtailment (EFLC), expected duration of load curtailment (EDLC), expected annual network losses (EANL) and the probability of load curtailment (PLC); and 2) those which describe each OHL performance separately, namely, the expected equivalent ageing index (EEAI), expected frequency of emergency loading (EFEL), expected duration of emergency loading (EDEL), expected magnitude of emergency loading (EMEL), expected annual line losses (EALL) and the probability of emergency loading (PEL). The mathematical formulations are detailed in D.

As shown in Fig. 1 all the calculations are performed iteratively until the analysis within a simulated year is completed, upon which a new simulation year is initiated by creating new operation and restoration histories of the network elements. The simulation is terminated when the covariance (cov) of the measured EENS index has reached a tolerance of $5 \%$ according to standard practice [16].

\section{B. System and Plant Data}

Reliability data describe the intact network topology of $X$ plants, including the normal failure $\lambda_{n-x}$ and repair $\mu_{n-x}$ rates based on their standard annual historical performance. The additional parameter, $\lambda_{e-y}$, is introduced in this methodology to determine the emergency failure rate of $y$ plant $\forall y \in X$ whilst operating at a critical elevated operating temperature due to a failure of a plant $x$ or an unfavorable severe change in weather and loading. The $\lambda_{e-y}$ parameter, therefore, is converted to the smallest $\Delta t$ defined in the system status initialization. Consequently, $\lambda_{e-y}$ accounts only for cascading events due to emergency elevated temperature operation during $\Delta t$, while $\lambda_{n-x}$ accounts for 'random' non-operational failures at the same $\Delta t$. This information can be provided from the network operator's historical data on cause of failure, failure and repair durations, and operating practice at emergency conditions.

Operational data, also available from network operators, specify the power system's operating limits. These include generator maximum power output, operating costs functions and technology types, transformer maximum power ratings, impedances, tap changing positions and synchronous condenser operating limits, circuit maximum normal/emergency ratings and impedances, as well as the annual load point chronological demand.

Weather data include hourly historical wind speeds, wind incidence angles, ambient temperatures and solar radiations that can be obtained from weather stations located close to OHLs or from installed DTR monitoring instrumentation.

OHL data describe the conductor technology, size, resistance, maximum strength, maximum permitted operating temperature as well as structure properties that define conductor installation tension and temperature, and design span length. This additional block of data is used to identify the conductor $I_{c, \max }(t)$ and $T_{c, \max }$, the maximum amount of ageing permitted by the design and to evaluate the occurred ageing due to contingency events. These data can be obtained from the OHL asset owners and conductor manufacturers.

\section{Network Status \& Constraint Mapping Computations}

Fig. 2 illustrates the computational steps implemented to calculate the updated network status \& constraint mapping at time $t$ as well as the next network status \& constraint mapping at the next time-step $t+\Delta t$ for all $X$ components. The updated network status accounts for any state transitions occurring at time $t$ due to thermal constraints and cascading events generated due to emergency operating conditions. The next network status includes only the updated transition state changes that occur at the next time-step $(t+\Delta t)$. More explicitly, the methodology considers four OHL thermal operational states: normal operation, pre-contingency high-loading, post-contingency highloading, and failure state; these are shown with distinct blue paths in Fig. 2. In order to identify each $x$ plant's operational state which indicates whether $\lambda_{n}$ or $\lambda_{e}$ is to be used, $T_{c, \max }$ is calculated with (2) and compared with the power flow at current transition time $T_{c}(t)$. Resultantly, for $T_{c, \max }>T_{c}(t), \lambda_{n}$ is used for plant $x$ whereas for $T_{c, \max }<T_{c}(t) \lambda_{e}$ is used instead as $x$ becomes a thermally constrained component $(y)$. This calculation is performed within the thermal constraint block for every identified high-loaded OHL component $y, \forall y \in X$ (Fig. 2).

\section{1) Normal Operation State}

The normal operation state (as illustrated by a blue/bold arrow in Fig. 2) assumes no plant failures and no thermal rating constraints due to weather or load changes. Consequently, the OHLs of the network are within their normal continuous thermal ratings and the sampling of the time to fail for every plant $x$, $\left(T T F_{n-x}\right)$, is computed using (3) considering that $\lambda_{n-x}$ follows an exponential failure distribution with $U_{n}$ (the unavailability of $x$ ) being a random number between 0 and 1 [16]. 


$$
T T F_{n-x}=-\left(1 / \lambda_{n-x}\right) \ln U_{n}
$$

When $T T F_{n-x}$ indicates a failure of OHL $x$ within the present $\Delta t$ time-step, then (4) is used to compute the repair time of plant $x,\left(T T R_{n-x}\right)$ which is stored within the next network status for the next $\Delta t$ duration calculations.

$$
T T R_{n-x}=-\left(1 / \mu_{n-x}\right) \ln U_{n}
$$

The normal operation transition history is evaluated at the beginning for all the components $x$ considering a year of operation segmented in sequential $\Delta t$ time-steps.

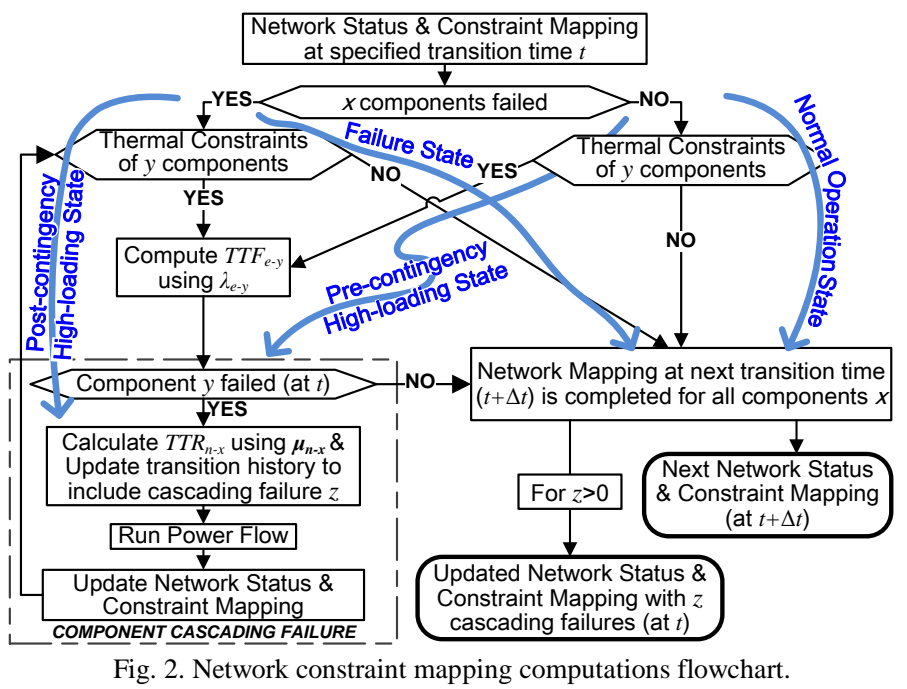

2) Pre-Contingency High-Loading State

Since the simulation considers weather and demand changes, it is then possible to model the additional state transitions that the network experiences caused by the weather or demand changes. Consequently the pre-contingency high-loading path shown in Fig. 2 represents the network's status when weather (or demand) transition leads to at least one of its $X$ OHLs to operate at emergency loading, even when no failures are present, resulting in a $T_{c, \max }<T_{c}(t)$ condition. In this case, $\lambda_{e-y}$, determines the transition status of component $y$, which is used to compute $T T F_{e-y}$ of $y$ with (5). $\lambda_{e-y}$ and $U_{y}$ are defined in reliability input data (Fig. 1).

$$
T T F_{e-y}=-\left(1 / \lambda_{e-y}\right) \ln U_{e}
$$

In (5), $\lambda_{e-y}$ is a function of the OHL's power flow and it is formulated assuming that the OHL failure rate increases exponentially with its loading, on condition that the OHL operates above its maximum continuous rating, $I_{c, \max }(t)$. The mathematical function for $\lambda_{e-y}$ is described by (6), where currents are in amperes. It can be seen from (6) that when the power flow of the line, $I_{c, f l o w}(t)$, is equal to its maximum normal rating then $\lambda_{e-y}=\lambda_{n}$, otherwise $\lambda_{e-y}<\lambda_{n}$ when the OHL operates within its emergency rating (i.e., $I_{c, f l o w}(t)>I_{c, \max }(t)$ ). (6) accounts for the exponential increase of conductor movement due to greater conductor sag (and reduced clearances) when operating at emergency rating. The coefficient $\alpha$ defines the OHL criticality index indicating the OHL's location (i.e., remote rural, slightly populated or urban areas) and the level of maintenance performed. The index is designed to vary from 1 to
5 (with 1 referring to frequent or recent maintenance) and is arbitrarily chosen due to lack of real data.

$$
\lambda_{e-y}=e^{\left(I_{c, f l o w}(t) / I_{c, \text { max }}(t)-1\right) \alpha} \cdot \lambda_{n-y}
$$

The unavailability of the high-loading line, $U_{e}$, is modeled as a function of power flow and maximum normal rating (in $\mathrm{A}$ ) using (7) to consider the increased unavailability that is expected at higher emergency loading operation. In (7) the coefficient $\beta$ indicates different operator acceptance risk levels due to different system active and standby redundancy levels (e.g. use of FACTS, fast generators, demand response). $\beta$ vary again from 1 to 5 with 5 referring to high risk (i.e., high redundancy) and 1 to low acceptance risk levels.

$$
U_{e}=1-U_{n}\left(I_{c, \max } / I_{c, \text { flow }}\right)^{\beta}
$$

This pre-contingency high-loading state considers the more realistic modeling of OHL behavior which enables identifying occasions when failure can be initiated from a component's high-loading operational state and subsequently lead to cascading failures within the power system.

The $T T F_{e-y}$ can determine if a cascading failure occurs at the same duration $\Delta t$ or a failure will occur at a future duration. When failure occurs at the same $\Delta t$ then the $T T R_{n-x}$ of the cascading failure $z$ is calculated and stored in the updated network status \& constraint mapping which is then used to identify any additional thermal constraints at this $\Delta t$ (feedback loop in Fig. 2). This considers plant cascading failure $z$ as an additional component $x$ in failure mode and that the failures at the updated network status have increased by $z(\forall z \in y)$. Then the process in Fig. 2 scans all network components $x$ to account for any new $y$ high-loaded lines. The feedback loop is repeated until all the $y$ plants that are in high-loading state are simulated and all possible cascading failures $z$ have been captured. Once all the $T T R_{n-x}$ for $z$ components are computed they are also stored in the next network status \& constraint mapping.

When $T T F_{e-y}$ indicates that a failure of a $y$ component occurs at a different $\Delta t$, then $T T F_{e-y}$ is stored in the next network status $\&$ constraint mapping and it is used in the next $\Delta t$ only when the component $y$ remains at high-loaded mode (due to the sequential nature of the analysis). If the loading or weather transitions result in converting the line to its normal loading then $T T F_{e-y}$ is cleared indicating that the emergency failure never occurred.

It should be noted that the components' repair/restoration time (in the modelling) is not affected by the nature of the failure and thus the $T T R_{n-x}$ is used to describe the repair times for both high and normal loadings.

\section{3) Post-Contingent High-Loading State}

The post-contingent high-loading state models the impact of high-temperature loadings on cascading failures. This is similar to pre-contingency high-loading state but differs in that the high-loading of a plant $y$ is the result of failure of another plant $x$. This state identifies plant failures that have influenced selected healthy lines to operate in high-loading state and therefore at increased failure risk. Consequently, this state models the likelihood of failure as a consequence of the decision to operate healthy lines at high temperatures for extensive durations in the desire to improve system adequacy 
during post-contingent events. In this operating state $T T F_{e-y}$ is also computed with (5) following the traversal path shown in Fig. 2. Finally, based on the resulting $T T F_{e-y}$ value, the $T T R_{n-x}$ is computed, when required, by using (4) and it updates network status at the same duration, $\Delta t$, as well as the next duration $t+\Delta t$.

\section{4) Component Failure State}

Once a plant $x$ has been identified as being in a failed state at duration $\Delta t$, the new $T T R_{n-x}$ of failed plant $x$ is computed according to (4). This then is stored in the next network status \& constraint mapping for the next computational time-step $t+\Delta t$.

\section{Network Optimization}

Network optimization computations are initiated by considering the updated network status \& constraint mapping (Fig. 3). This contains the updated network status (including $z$ cascading failures) produced from the computations in Fig. 2 (i.e., the values for the altered network admittance matrix and the subsequent power flow results) as well as the network operational data (shown in Fig. 1). The updated network status is scanned for violations of thermal and voltage constraints. When no constrains are identified then no optimization is performed as the network is operating at its optimal state (Fig. $3)$. In this case, no update of the network and OHL indices occurs and the Network \& OHL state reliability mapping remains unaltered at this time-step (Fig. 1).

However, when constraint violations are identified, network optimization is performed driven by three different objective functions that can be used either in combination or individually. These are the minimizations of: (i) the conventional generation and load curtailment cost, $C_{j} \cdot G_{j}$, (ii) the proposed OHL losses cost, $C_{x} \cdot P_{x}$, and (iii) the ageing cost, $C_{x} \cdot E E A I_{x}$ (Fig. 3). The inclusion of electro-thermal modeling helps to capture the losses and ageing of each OHL and consequently allow for their optimization based on specific OHLs or the complete network. In addition, the implemented electro-thermal modeling computes more accurately OHL power losses as it accounts for the effect of conductor temperature on its resistance. The objective functions are minimized subject to voltage (KVL), current flow (KCL), generation apparent power at a particular time $G_{M A X}(t)$ and OHL maximum continuous apparent power at a particular time $S_{M A X}(t)$ constraints.

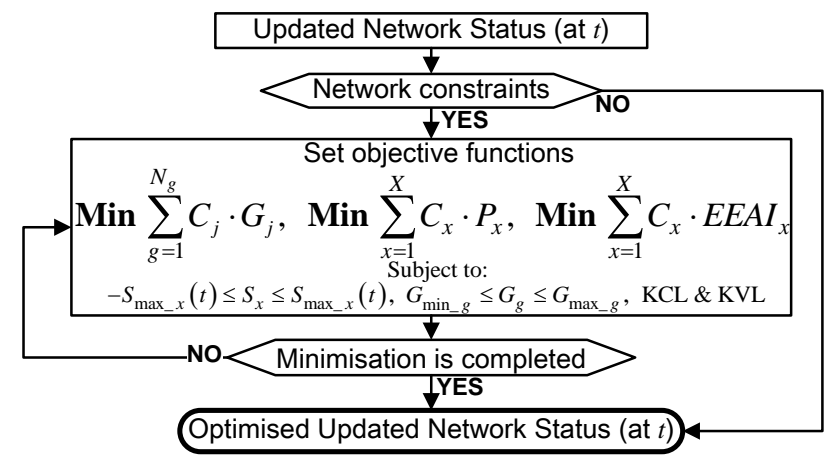

Fig. 3. Flowchart of the network optimization procedure.

An iteration loop is used for the minimization of the objective functions (for $N_{g}$ generators and $X$ lines) with the network \& OHL state reliability mapping block as shown in Fig.
1 in order to calculate both network and OHL performance indices. This process is necessary for the $C_{x} \cdot P_{x}$ and $C_{x} \cdot E E A I_{x}$ as their optimization is solvable only through the iterative benders decomposition [32]. The $C_{j} \cdot G_{j}$ is solvable through simpler quadratic or linear programming techniques.

Once the minimization iteration is completed for the current $\Delta t$ time-step the updated system and OHL indices are stored within the optimized updated network status (in Fig. 3), which are updated in every $\Delta t$ loop until the completion of the year.

\section{1) Network Performance Indices}

System optimization is driven by the need to evaluate network performance indices. Therefore, EDLC, PLC, EFLC and EENS are evaluated based on (8)-(11), where, $d_{j, i}$ is the duration of load curtailment of the $j^{\text {th }}$ system interruption in year $i, N_{i}$ is the number of load curtailment occurrences, SysENS $S_{j, i}$ is the system energy not supplied (in MWh) for the $j^{\text {th }}$ interruption in year $i$ and $T_{s}$ is the total simulation time, in years.

$$
\begin{gathered}
E D L C=\sum_{i=1}^{N}\left(\sum_{j=1}^{M} d_{j, i}\right) / T_{s} \\
P L C=E D L C / 8760 \\
E F L C=\sum_{i=1}^{M} N_{i} / T_{s} \\
E E N S=\sum_{i=1}^{N}\left(\sum_{j=1}^{M} S_{s y S} E N S_{j, i}\right) / T_{s}
\end{gathered}
$$

\section{2) OHL Performance Indices}

The $C_{x} \cdot P_{x}$ and $C_{x} \cdot E E A I_{x}$ costs are computed in a single stage when the optimization targets $C_{j} \cdot G_{j}$. However, when the optimization targets $C_{x} \cdot P_{x}$ and $C_{x} \cdot E E A I_{x}$ costs, the OHL indices are computed in two stages as bender cuts. In the first stage the EDEL, PEL, EFEL and EMEL are calculated based on (12)-(15) for the $\Delta t$ time-step of the simulation.

$$
\begin{gathered}
E D E L=\sum_{i=1}^{N}\left(\sum_{j=1}^{M} d_{-} \text {eme }_{j, i}\right) / T_{s} \\
P E L=E D E L / 8760 \\
E F E L=\sum_{i=1}^{M} N_{-} \text {eme } / T_{s} \\
E M E L=\sum_{i=1}^{N}\left(\sum_{j=1}^{M} \text { LineOverload }_{j, i}\right) / T_{s}
\end{gathered}
$$

Where, $d \_$eme $_{j, i}$ is the emergency loading duration of the plant of the $j^{\text {th }}$ system interruption in year $i, N$ eme is the number of emergency loading events, and LineOverload ${ }_{j, i}$ is the emergency loading magnitude for the $j^{\text {th }}$ interruption in simulation year $i$.

In the second stage, EMEL along with the OHL design and the optimized updated network status data are used in (2) to compute conductor temperature, $T_{c}(t)$, stress, $\sigma_{x, T c}$, and thermal rating, $T_{c, \max }(t)$, for each OHL at $\Delta t$ (Fig. 4). The $T_{c}(t)$ and conductor resistance, $R_{x}\left(T_{c}(\Delta t)\right)$, are used to compute the $E A L L_{x}$ for each OHL, $x$, by using (16). $\operatorname{LineLoad}_{x, i}(\Delta t)$ is the loading of plant $x$ during $\Delta t$ in simulation year $i$. Finally, $E A N L_{x}$ that describes overall network losses is calculated using (17) completing the OHL losses mapping at $t$. 


$$
\begin{gathered}
E A L L_{x}=\sum_{i=1}^{N}\left(\left(\text { LineLoad }_{j, i}\right)^{2} \times R_{x}\left(T_{c}(\Delta t)\right)\right) \\
E A N L=\sum_{x=1}^{N_{L n}} E^{\prime} L_{x}
\end{gathered}
$$

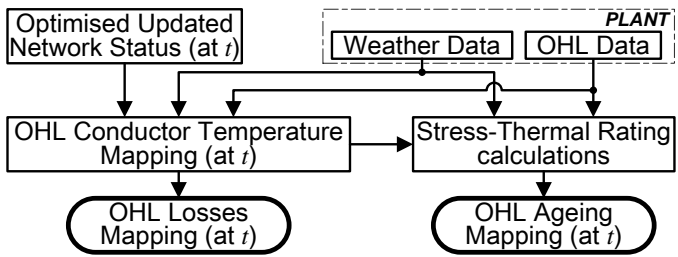

Fig. 4. Calculation of OHL reliability indices mapping flowchart

The $E E A I_{x}$ of each OHL at the same time is calculated last to create the OHL ageing mapping (Fig. 4). For this step an additional OHL computation is implemented using $T_{c}(t)$, $T_{c, \max }(t)$ and $\sigma_{x, T c}$ based on methods discussed in [31, 33] to capture the elevated conductor temperature operation ageing. This could accelerate conductor ageing, reduce conductor life and affect OHL ground clearances (i.e., OHL design reliability).

Different conductor technologies exhibit different ageing characteristics which are described by different equations. This occurs at operating temperatures above $95^{\circ} \mathrm{C}$ for Aluminum Conductors Steel Reinforced (ACSRs) with high steel content and above $75^{\circ} \mathrm{C}$ for ACSRs with low steel content or aluminum conductors [33]. The ageing modelling is implemented by using (18) which is adopted from [33] to quantify the elevated conductor ageing $\varepsilon_{x, T c, t}$ of an OHL, $x$, operating at $T_{c}$ for a duration $t_{x, T c}, \varepsilon_{x, T c, t}$ is affected by conductor type $K_{x}$, installation stress $\sigma_{x, T c}$, and the duration $t_{x, T_{c}}$ of operation at $T_{c}$ [33].

$$
\varepsilon_{x, T_{C}, t}=f\left(K_{x}, T_{c}, \sigma_{x, T_{C}}, t_{x, T_{C}}^{0.16}\right)
$$

From (18) the calculated ageing mapping is segmented at different $T_{c}$ and durations $t_{x, T c}$ based on the OHL operating profile, which varies for each OHL. In order to generate a comparative quantity of ageing between the different OHLs the equivalent ageing at $100^{\circ} \mathrm{C}, \varepsilon_{x, 100}$ is calculated at an annualized base by using (19). Consequently, (20) is produced to calculate the $E E A I_{x}$ at $100^{\circ} \mathrm{C}$, in hours, for an OHL $x$ that has experienced a number of elevated temperature events $(E T E)$ at various elevated temperatures $T_{c}$ and durations $t_{x, T_{c}}$ within a year.

$$
\begin{gathered}
f\left(K_{x}, T_{c}(t), \sigma_{x, T_{C}}, t_{x, T_{C}}^{0.16}\right)=f\left(K_{x}, 100^{\circ} C, \sigma_{x, 100}, t_{x, 100}^{0.16}\right) \\
E E A I_{x}=\sum_{i=1}^{E T E} t_{x, 100}=\sum_{i=1}^{E T E}\left(\frac{e_{x, T_{C}}}{e_{x, 100}} \times t_{x, T_{C}}^{0.16}\right)^{6.25}
\end{gathered}
$$

\section{APPLICATION OF THE METHODOLOGY}

The methodology is applied to IEEE-RTS 96 considering the data provided in [34] with $1.5 \mathrm{pu}$ increase in demand to stress the system and encounter the different OHL emergency loadings and network states (Fig. 2). Only line failures are considered while generators are $100 \%$ reliable with their capacity evenly increased to $2 \mathrm{pu}$.

\section{A. Modelling of Emergency Failures}

The newly introduced $\lambda_{e}$ is not provided in [34] and thus different scenarios, shown in Table I, are assumed to examine the impact of $T T F_{e-y}$ on network performance. Sc-1 is the base case, while Sc-2 considers $\lambda_{e}$ as a multiple of $\lambda_{n}$ with two cases capturing the (medium and high) risk of OHL failure during high-loading operation. Sc-3 models $\lambda_{e}$ as a function of OHL loading with different criticality indices and Sc-4 advances further the modeling of Sc-3 by including OHL unavailability as a function of OHL operation risk acceptance. Three operation risk acceptance values are used to indicate three levels of active network redundancy the operator might wish to consider.

\section{B. OHL Plant Data Assumptions}

OHL design data are not provided in [34], thus assumptions are made for both OHL design and ambient weather data.

\section{1) OHL Design Properties Assessed}

Table II shows the aluminum conductor sizes which were carefully selected so as to provide similar current ratings as in [34] but also realistic thermal ratings [35]. These ratings are used for both ageing and power flow calculations and are based on $20^{\circ} \mathrm{C}$ ambient temperature, $0.5 \mathrm{~m} / \mathrm{s}$ wind speed and nil solar radiation [35]. Conductor ageing calculations also consider an average OHL span of $400 \mathrm{~m}$ for the $230 \mathrm{kV}$ and $300 \mathrm{~m}$ for the

\begin{tabular}{|c|c|c|}
\hline & nario 1 (Sc-1): Base case & $\lambda_{e}=\lambda_{n}, \alpha=0, U_{e}=U_{n}, \beta=0$ \\
\hline & Scenario 2 (Sc-2): $\lambda_{e}=$ constant & $\begin{array}{c}\lambda_{e}=k \lambda_{n}, k=2.5,3.5 \\
U_{e}=U_{n}, \beta=0\end{array}$ \\
\hline 䓠 & Scenario 3 (Sc-3): $\lambda_{e}=$ from (6) & $\begin{array}{c}\text { criticality index values } \alpha=1,3,5 \\
U_{e}=U_{n}, \beta=0\end{array}$ \\
\hline$\left.\frac{1}{.00}\right]$ & $\begin{array}{c}\text { Scenario } 4(\mathrm{Sc}-4): \lambda_{e}=\text { from }(6) \\
\text { and } U_{e} \text { from (7) }\end{array}$ & $\begin{array}{c}\text { criticality index values } \alpha=1,3,5 \\
\text { risk index values } \beta=1,3,5\end{array}$ \\
\hline
\end{tabular}
$138 \mathrm{kV}$ systems with a $20 \%$ conductor rated breaking strength installation tension at $5^{\circ} \mathrm{C}$ temperature [36].

TABLE I

MODELLING SCENARIOS AND ASSUMPTIONS OF EMERGENCY OHL FAILURES

TABLE II

CONDUCTOR PROPERTIES AND RATINGS MODELED IN IEEE-RTS 96 NETWORK

\begin{tabular}{c|c|c|c|c}
\hline Name & $\mathbf{R}_{\mathbf{A C}}(\mathbf{\Omega} / \mathbf{k m})$ & $\mathbf{I}_{\text {Norm }}(\mathbf{A})$ & $\mathbf{I}_{\text {EM-Long }}(\mathbf{A})$ & $\mathbf{I}_{\text {EM-Short }}(\mathbf{A})$ \\
\hline $\begin{array}{c}\text { Upas } \\
(138 \mathrm{kV})\end{array}$ & 0.09396 at $25^{\circ} \mathrm{C}$ & $732\left[60^{\circ} \mathrm{C}\right]$ & $873\left[79^{\circ} \mathrm{C}\right]$ & $923\left[87^{\circ} \mathrm{C}\right]$ \\
\hline $\begin{array}{c}\text { Araucaria } \\
(230 \mathrm{kV})\end{array}$ & 0.04266 at $25^{\circ} \mathrm{C}$ & $1272\left[63^{\circ} \mathrm{C}\right]$ & $1503\left[82^{\circ} \mathrm{C}\right]$ & $1567\left[88^{\circ} \mathrm{C}\right]$ \\
\hline
\end{tabular}

\section{2) Weather Data}

The calculations of conductor temperature profiles required for the probability of exceeding OHL design thermal ratings are based on wind, ambient temperature and solar radiation measurements from a real DTR (CAT-1) monitoring system installed on a National Grid's lattice tower.

\section{Design-Operation Network Assessment}

The Network is assessed based on the proposed methodology at two different stages: at OHL design performance and at overall network performance. This, therefore, allows incorporating the outputs from the network evaluation to optimize OHL design and then further improve the network in a more holistic design-operation perspective. The outputs of the analyses are produced using SMC simulation for 1500 years with a $5 \%$ cov.

\section{1) Assessment of OHL design}

From the OHL designed thermal rating values (Table II) and the provided weather data, the excursion times for each current 
rating in Table II (for both voltages) are calculated and the outputs are shown in Fig. 5 ('+' symbol). These excursions are produced under the assumption of a continuous loading at the design current rating throughout the year and indicate the risk (probability) of exceeding the corresponding design temperature curves shown in Fig. 5. Both OHLs have similar excursions, indicating realistic design assumptions are made (Fig. 5).

Fig. 5 also shows the excursion probability curves (dotted lines) for conductor ageing temperatures $\left(75^{\circ} \mathrm{C}\right)$. These indicate that the risk of ageing included in the OHL design for each (normal, emergency) current rating vary from $0.45 \%$ to $24 \%$ for Upas and from $1 \%$ to $24 \%$ for Araucaria with the higher values denoting the emergency operation. The normal rating excursions indicate negligible (up to 1\%) design risk which is in agreement with [35] while the emergency rating excursions indicate higher (up to $24 \%$ ) design risk of conductor ageing at the OHL design stage. The risk of ageing in these OHL designs can be further quantified from the conductor operating temperature frequencies in Fig. 6, showing the maximum expected operating temperature, the operating hours at each temperature and the excursions (shaded areas) for the current ratings in Table II.

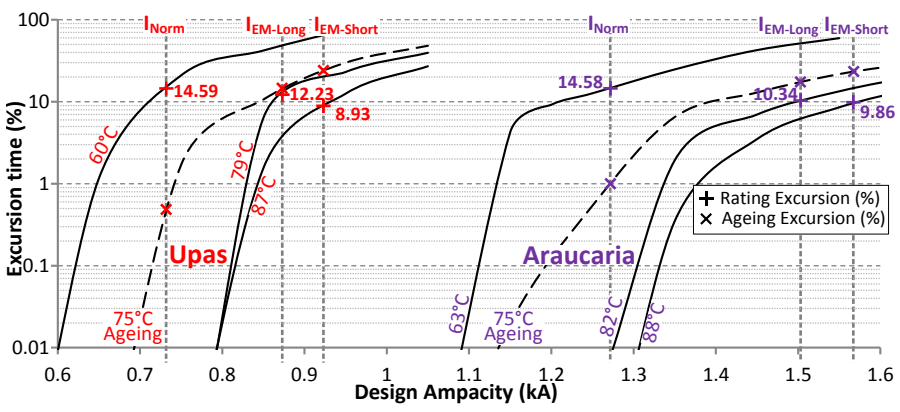

Fig. 5. Excursion times for Upas and Araucaria OHL designs.

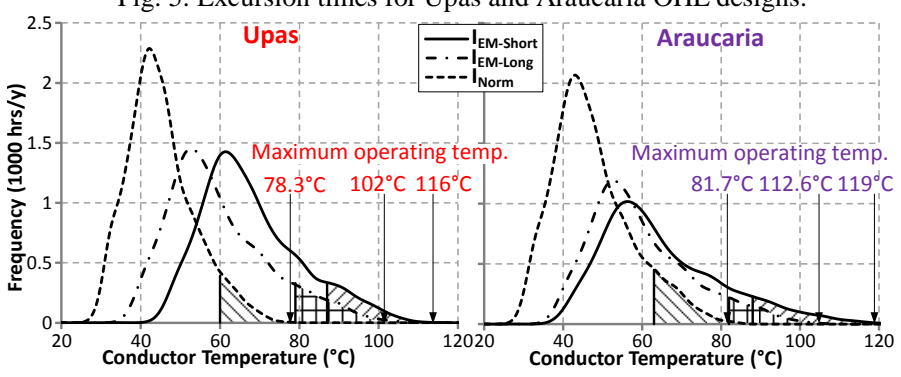

Fig. 6. Temperature distributions for $138 \mathrm{kV}$ and $230 \mathrm{kV}$ OHL designs.

When the complete network performance is considered for the evaluation of its 32 OHLs (among the 38 branch components), different ageing values are computed for each line, identifying lines 12, 23 and 28 as the most "critical". These lines are affected considerably more than others (e.g., line 13 which is the next 'critical'), as shown in Table III, indicating therefore that OHL rating design based on excursions as suggested in [35] does not represent the same risk of ageing for all OHLs in the network. Thus, excursion alone cannot be used to quantify the actual OHL design risk.

The comparison of the EEAI and EALL for lines 12, 13, 23 and 28 at the different modelled scenarios (Table III) shows that the increase in emergency failure rate reduces the expected ageing of the specific lines as well as their annual losses. This is due to reduced operation of the lines at emergency state as more failures are expected to occur when an OHL is high-loaded. This can also be observed more clearly from the EDEL values shown in Table III.

The recorded EDEL values indicate the duration of operating the critical lines at emergency range temperatures. The values for $\mathrm{Sc}-1$ are the largest among the different modelling scenarios as the OHLs under this scenario have emergency failure rate equal to normal. When an increased risk of failure is considered at the emergency operation (Sc-2 to Sc-4) then reduced EDEL durations are expected due to the increased number of failures (when lines operate at their emergency loading). Consequently, a large EDEL value can be translated into an increased risk of conductor accelerated ageing due to prolonged elevated temperature operation. However, EDEL values are not linearly correlated with ageing as the EDEL is composed of multiple emergency operation events of different durations at different times. Therefore, a single hour event at very high temperature (e.g. maximum $116^{\circ} \mathrm{C}$ in Fig. 6) could lead to a larger EEAI value than a multiple hour event at a temperature just above the $75^{\circ} \mathrm{C}$ threshold. EDEL values can, thus, be used to indicate the usefulness of implementation of emergency ratings of individual lines while EEAI values indicate the severity of the OHL loadability (resulting from emergency rating) on its long-term life.

TABLE III

SUMMARY OF LINE BASED PERFORMANCE INDICES

\begin{tabular}{c|c|c|c|c|c|c|c|c|c}
\hline Ln & Sc-1 & \multicolumn{2}{|c|}{ Sc-2 } & \multicolumn{4}{c|}{ Sc-3 } & \multicolumn{3}{c}{ Sc-4 } \\
\hline & $\alpha, \beta=0$ & $k=2.5$ & $k=3.5$ & $\alpha=1$ & $\alpha=3$ & $\alpha=5$ & $\alpha, \beta=1$ & $\alpha, \beta=3$ & $\alpha, \beta=5$ \\
\hline & \multicolumn{6}{|c|}{ Expected Equivalent } & Ageing Index $-E E A I(\mathrm{~h} / \mathrm{year})$ \\
\hline 12 & 4.66 & 1.13 & 0.78 & 1.83 & 0.39 & 0.17 & 0.39 & 0.08 & 0.04 \\
\hline 13 & 0.26 & 0.06 & 0.04 & 0.10 & 0.02 & 0.01 & 0.02 & 0.00 & 0.00 \\
\hline 23 & 14.7 & 3.55 & 2.47 & 5.77 & 1.24 & 0.53 & 1.23 & 0.26 & 0.11 \\
\hline 28 & 16.6 & 4.01 & 2.78 & 6.51 & 1.40 & 0.59 & 1.39 & 0.30 & 0.13
\end{tabular}

Expected Annual Line Losses - EALL (GWh/year)

\begin{tabular}{r|r|r|r|r|r|r|r|r|r}
\hline 12 & 9.75 & 7.70 & 6.96 & 8.97 & 8.48 & 7.41 & 8.09 & 6.63 & 6.23 \\
\hline 13 & 1.75 & 1.38 & 1.25 & 1.61 & 1.52 & 1.33 & 1.46 & 1.19 & 1.12 \\
\hline 23 & 29.89 & 23.62 & 21.34 & 27.50 & 26.00 & 22.72 & 24.81 & 20.32 & 19.13 \\
\hline 28 & 10.21 & 8.06 & 7.29 & 9.39 & 8.88 & 7.76 & 8.47 & 6.94 & 6.53 \\
\hline & \multicolumn{8}{c}{ Expected Duration of Emergency Loading - EDEL (h/year) } \\
\hline 12 & 32.1 & 7.78 & 5.37 & 12.6 & 2.68 & 1.17 & 2.68 & 0.55 & 0.27 \\
\hline 13 & 7.65 & 1.76 & 1.17 & 2.94 & 0.58 & 0.29 & 0.58 & 0 & 0 \\
\hline 23 & 95.5 & 54.9 & 38.2 & 65.6 & 19.2 & 8.19 & 19.1 & 4.02 & 1.70 \\
\hline 28 & 108.6 & 69.3 & 48.0 & 86.9 & 24.1 & 10.1 & 24.0 & 5.18 & 2.24 \\
\hline \multicolumn{10}{c}{ TABLE IV }
\end{tabular}

SUMMARY OF SYSTEM BASED PERFORMANCE INDICES

\begin{tabular}{l|c|r|r|r|r|r|r}
\hline & & $\begin{array}{c}\text { EENS } \\
\text { GWh/yr }\end{array}$ & $\begin{array}{c}\text { EFLC } \\
\text { Occ/yr }\end{array}$ & $\begin{array}{c}\text { EDLC } \\
\mathrm{h} / \mathrm{yr}\end{array}$ & $\begin{array}{c}\text { EIC } \\
\mathrm{M} \$\end{array}$ & $\begin{array}{c}\text { EANL } \\
\text { GWh/yr }\end{array}$ & $\begin{array}{c}\text { EEAI } \\
\mathrm{h} / \mathrm{yr}\end{array}$ \\
\hline Sc-1 & $\alpha=\beta=0$ & 2.50 & 2.65 & 6.87 & 2.74 & 132.87 & 38.65 \\
\hline \multirow{2}{*}{ Sc-2 } & $k=2.5$ & 10.34 & 7.01 & 15.20 & 31.38 & 104.9 & 10.85 \\
\cline { 2 - 8 } & $k=3.5$ & 14.89 & 11.32 & 19.53 & 38.49 & 4.87 & 8.15 \\
\hline \multirow{3}{*}{ Sc-3 } & $\alpha=1$ & 6.37 & 4.98 & 11.05 & 5.01 & 122.33 & 16.37 \\
\cline { 2 - 8 } & $\alpha=3$ & 7.15 & 6.01 & 12.16 & 5.32 & 115.61 & 14.80 \\
\cline { 2 - 8 } Sc-4 & $\alpha=5$ & 11.73 & 8.53 & 17.63 & 33.68 & 100.94 & 9.80 \\
\cline { 2 - 8 } & $\alpha=\beta=1$ & 7.38 & 6.18 & 12.92 & 5.43 & 110.36 & 14.40 \\
\cline { 2 - 8 } & $\alpha=\beta=3$ & 19.94 & 17.54 & 22.50 & 53.29 & 90.35 & 6.59 \\
\hline
\end{tabular}

2) Assessment of the Network Wide Performance

The computed outputs for the overall network performance are shown in Table IV with the energy interruption cost (EIC) being calculated based on the curtailment duration and sector type as in [16]. The results in Table IV indicate a reduced network performance when increased rate of failures at emergency operation is considered in the modeling. In 
particular, Sc-1 $\left(\lambda_{e}=\lambda_{n}\right)$ results in the lowest EENS and EIC compared to all other scenarios, while it develops the most OHL network ageing. This ageing should in reality have an impact both on the failure rate at emergency loading (since the initial OHL clearances are reduced), and on the overall network operation costs (since increased maintenance costs are required). Neither ageing and increased risk of failures at emergency conditions nor their associated increased operation costs are captured with current methodologies. They could, thus, falsely indicate that extensive durations at emergency operating conditions result in improved network performance due to increased operational flexibility, since they do not capture the increased operation risk and asset ageing associated with this action. This limitation is overcome with the proposed methodology which captures the risk of increased durations at emergency loading operation and increased excursions.

The results in Table IV suggest that increased durations of emergency loading (e.g. 24 hours), without considering the impact of conductor ageing and increased operation risk from the reduced clearances, do not capture the total operating network costs since the increased ageing on conductors will lead to more frequent failures and/or increased maintenance costs. Furthermore current methods do not capture the actual risk of cascading failures. In this example, the comparison of single failure events and cascading failures for the network under the base Sc-1 and the Sc-2 $(k=2.5)$ is presented in Table V. This comparison illustrates that when an increased failure rate is considered for the high-loading OHL operation, to capture the failure risk due to increased conductor sag, the number of single failure events, expected to occur in a year, is approximately doubled due to their operation at emergency loading. This, in turn, results in increased loading of the remaining constrained network and consequently in an increased number of post contingency high-loading events as it can be derived from the increased number of double, triple and multiple cascading events in Table V when Sc-2 is implemented.

The very large observed increase in double failures (compared to the triple or more than triple events) is purely due to the result of a single failure making a neighbor OHL to operate at high-loading state and consequently increasing the probability of failure resulting in cascading failure. However, for the examined network topology when a second cascading failure occurs the network results in load shedding and increased EENS as shown in Table IV. This increase in EENS reduces therefore the number of triple (or more) failures compared to the double signifying that the IEEE-RTS 96 network is not very "strong" in triple failures. Consequently, the study of high-loading cascading events can be used to identify the resilience of a network on emergency operations.

$$
\text { TABLE V }
$$

NUMBER OF SYSTEM'S OHL FAILURE EVENTS IN ANNUAL BASIS (OCC/YR)

\begin{tabular}{|c|c|c|c|c|}
\hline Scenario & Single & Double & Triple & More \\
\hline Sc-1 & 62.63 & 3.86 & 2.754 & 0.074 \\
\hline Sc-2 $(k=2.5)$ & 126.52 & 28.58 & 6.348 & 3.6 \\
\hline
\end{tabular}

It should be noted that the absence of metrics to correlate the failures with component loading makes it difficult to directly compare the results from Sc-2 to Sc-4, and thus to conclude which one captures more accurately the actual network emergency loading risks. However, Sc-4 is more advanced since it allows for different line criticality and network flexibility levels to be modelled for each OHL or network sections.

\section{CONCLUSIONS}

A new framework for power system reliability assessment is presented in this paper. It incorporates the electro-thermal status of OHL network in reliability assessment and thus improves the existing reliability assessment methods as it captures the increased risk of failures of components when operating for extensive durations at emergency ratings. Consequently, it can help evaluate a network's resilience in emergency operation events.

In addition, it allows the inclusion of OHL ageing and associated costs due to increased risk (through higher excursion times) considered at OHL design level. This methodological extension could enable more informed decisions to be made at network (planning and operation) level and allows considering the risk-based cost of increased exceedances from utilizing OHL network assets at higher thermal ratings. For example, when interruption costs are high this could support increased OHL thermal ratings through increased excursions of PTR which could justify the increased OHL ageing (life reduction) risk and maintenance costs.

The increased visibility of network OHL assets afforded with the proposed indices can provide insights on assessment of ageing and maintenance costs of critical OHLs against network performance improvements through extended emergency durations or 'ageing free' conductor technologies. Furthermore, 'expensive' investments for ageing mitigating solutions to reduce failure risk can under this assumption be justified.

The proposed methodology will be further expanded in future work to include the electro-thermal modelling of underground cable systems that capture both cable properties and ducting systems, in combination with the soil (environmental) conditions they are installed in. This would help identify the critical cables within a network based on ageing. This identification along with the currently established approach for modelling OHL's ageing will allow utilities to take more comprehensively informed decisions on policies for optimal cable or OHL replacement and emergency rating levels.

\section{REFERENCES}

[1] "Increased Power Flow Guide Book: Increasing Power Flow on Transmission and Substation Circuits," EPRI, Palo Alto, 2005.

[2] R. F. Chu, "On selecting transmission lines for dynamic thermal line rating system implementation," IEEE Transactions on Power Systems, vol. 7, pp. 612-619, 1992.

[3] R. Stephen, "Decsription and Evaluation of Options Relating to Uprating of Overhead Transmission Lines," Paris, 2004.

[4] H. Wan, J. D. McCalley, and V. Vittal, "Increasing thermal rating by risk analysis," IEEE Trans. on Power Systems, vol. 14, pp. 815-828, 1999.

[5] S.P. Hoffmann and A.M. Clark, "The Approach to Thermal Uprating of Transmission Lines in the UK," in CIGRE Session, Paper B2-317, 2004. 
[6] C. F. Price and R. R. Gibbon, "Statistical Approach to Thermal Rating of Overhead Lines For Power Transmission And Distribution," IEE Proceedings C: Generation Transmission and Distribution, vol. 130, pp. 245-256, 1983.

[7] M. Monseu, "Determination of thermal line ratings from a probabilistic approach," in Third International Conference on Probabilistic Methods Applied to Electric Power Systems, 1991, pp. 180-184.

[8] H. Shaker, M. Fotuhi-Firuzabad, and F. Aminifar, "Fuzzy Dynamic Thermal Rating of Transmission Lines," IEEE Transactions on Power Delivery, vol. 27, pp. 1885-1892.

[9] D. M. Greenwood, J. P. Gentle, K. S. Myers, P. J. Davison, I. J. West, J. W. Bush, et al., "A Comparison of Real-Time Thermal Rating Systems in the U.S. and the U.K," IEEE Transactions on Power Delivery, vol. 29, pp. 1849-1858, 2014.

[10] D. A. Douglass and A. Edris, "Real-time monitoring and dynamic thermal rating of power transmission circuits," IEEE Transactions on Power Delivery, vol. 11, pp. 1407-1418, 1996.

[11] M. J. Tunstall, S. P. Hoffmann, N. S. Derbyshire, and M. J. Pyke, "Maximising the ratings of National Grid's existing transmission lines using high temperatures, low sag conductor," in CIGRE Session, Paper 22202, Paris France, Aug. 2000.

[12] M. Tunstall, N. Derbyshire, S. Hoffmann, and M. Pyke. United Kingdom. "Assessment of $620 \mathrm{~mm}^{2}$ 'Matthew' GZTACSR Gap Type Conductor for use on L2 Towers," National Grid, Technical Report TR(E)327, 1999.

[13] F. D. Pullen and D. J. Hopgood, "Probabilistic approaches to transmission line thermal ratings," in Third International Conference on Probabilistic Methods Applied to Electric Power Systems, 1991, pp. 191-196.

[14] P. Pourbeik, Kundur, P. S., Taylor, C. W., "The Anatomy of a Power Grid Blackout - Root Causes and Dynamics of Recent Major Blackouts," IEEE Power and Energy Magazine, vol. 4, pp. 22-29, 2006.

[15] Cigre Working Group B2.20, "Management of Risks Due to Load-Flow Increases in Transmission OHL," Cigre broshure 385, June 2009.

[16] R. Billinton and W. Li, Reliability Assessment of Electrical Power Systems Using Monte Carlo Methods. London: Springer, 1994

[17] U. G. Knight, Power Systems in Emergencies: From Contingency Planning to Crisis Management: John Wiley \& Sons, 1994.

[18] R. Billinton, "Bibliography on the Application of Probability Methods In Power System Reliability Evaluation," IEEE transactions on Power apparatus and systems, vol. PAS-91, pp. 649-660, 1972.

[19] R. N. Allan, R. Billinton, and S. H. Lee, "Bibliography of the Application of Probability Methods in Power System Reliability Evaluation 1977 1982," IEEE Power Engineering Review, vol. PER-4, pp. 24-25, 1984.

[20] M. T. Schilling, A. M. Leite da Silva, R. Billinton, and M. A. El-Kady, "Bibliography on power system probabilistic analysis (1962-88)," IEEE Transactions on Power Systems, vol. 5, pp. 1-11, 1990.

[21] R. N. Allan, R. Billinton, A. M. Breipohl, and C. H. Grigg, "Bibliography on the application of probability methods in power system reliability evaluation: 1987-1991," IEEE Transactions on Power Systems, vol. 9, pp. 41-49, 1994.

[22] R. Billinton, M. Fotuhi-Firuzabad, and L. Bertling, "Bibliography on the Application of Probability Methods in Power System Reliability Evaluation 1996-1999," IEEE Power Engineering Review, vol. 21, pp. 56$56,2001$.

[23] R. Billinton and W. Peng, "Teaching distribution system reliability evaluation using Monte Carlo simulation," IEEE Transactions on Power Systems, vol. 14, pp. 397-403, 1999.

[24] R. Billinton and W. Wangdee, "Impact of utilising sequential and nonsequential simulation techniques in bulk-electric-system reliability assessment," IEE Proceedings Generation, Transmission and Distribution, vol. 152, pp. 623-628, 2005.

[25] A. M. Rei, M. T. Schilling, and A. C. G. Melo, "Monte Carlo Simulation and Contingency Enumeration in Bulk Power Systems Reliability Assessment," in International Conference on Probabilistic Methods Applied to Power Systems, 2006, pp. 1-6.

[26] D. M. Greenwood and P. C. Taylor, "Investigating the Impact of RealTime Thermal Ratings on Power Network Reliability," IEEE Transactions on Power Systems, vol. PP, pp. 1-9, 2014.

[27] H. Shaker, H. Zareipour, and M. Fotuhi-Firuzabad, "Reliability Modeling of Dynamic Thermal Rating," IEEE Transactions on Power Delivery, vol. 28, pp. 1600-1609, 2013.

[28] National Grid Electricity Transmission. (March 2015). Electricity Ten Year Statement 2014 [Online] Available:
http://www2.nationalgrid.com/UK/Industry-information/Future-ofEnergy/Electricity-Ten-Year-Statement/

[29]D. O. Koval and R. Billinton, "Determination of Transmission Line Ampacities by Probability and Numerical Methods," IEEE Transactions on Power apparatus and systems, vol. PAS-89, pp. 1485-1492, 1970.

[30] "IEEE Standard for Calculating the Current-Temperature of Bare Overhead Conductors," IEEE Std. 738-2006, pp. 1-59, 2007.

[31] "IEEE Guide for Determining the Effects of High-Temperature Operation on Conductors, Connectors, and Accessories," IEEE Std. 1283-2004, pp. 1-28, 2005

[32] G. Strbac, S. Ahmed, D. Kirschen, and R. Allan, "A method for computing the value of corrective security," IEEE Transactions on Power Systems, vol. 13, pp. 1096-1102, 1998.

[33] K. Kopsidas, S. M. Rowland, and B. Boumecid, "A Holistic Method for Conductor Ampacity and Sag Computation on an OHL Structure," IEEE Transactions on Power Delivery, vol. 27, pp. 1047-1054, 2012.

[34] P. M. Subcommittee, "IEEE Reliability Test System," IEEE Transactions on Power Apparatus and Systems, vol. PAS-98, pp. 2047-2054, 1979.

[35] "Engineering Recomendation P27: Current rating guide for high voltage overhead lines operating in teh UK distribution system," Energy Network Association, 1986.

[36] K. Kopsidas, "Assessing the power transfer performance of a lattice tower overhead line system," IET Generation, Transmission \& Distribution, vol. 7, pp. 90-100, 2013.

\section{BIOGRAPHIES}

Konstantinos Kopsidas (M'06) received a BEng in Electrical Engineering from the Institute of Piraeus, Athens, Greece in 2002, a Class I BEng in Electrical and Electronic Engineering from The University of Manchester Institute of Science and Technology (UMIST), U.K., in 2004, an MSc (with distinction) in 2005 and a PhD in 2009 in Electrical Power Engineering from The University of Manchester. Since 2011, he is a Lecturer with the School of Electrical and Electronic Engineering in The University of Manchester with main research interests on plant modeling and reliability and adequacy.

Chomba Tumelo-Chakonta (S' 10) completed the BEng, MSc and PhD degrees all in Electrical and Electronic Engineering at the University of Manchester from 2005 to 2015. Chomba's research interests reside within the broader topics of power system reliability \& asset management, with a current emphasis on tackling the problems related to increasing the power flows of existing power networks.

Carlos Cruzat (S' 15) obtained his BEng in Electrical Engineer from the University of Concepción, Chile and his MSc from the Imperial College London. $\mathrm{He}$ is currently a $\mathrm{PhD}$ student at the University of Manchester specializing in reliability of power systems. Prior to his $\mathrm{PhD}$ he worked as Electrical Engineer in Mining Company BHP Billiton for 5 years. 